\title{
Living with a giant, flowering parasite: metabolic differences between Tetrastigma loheri Gagnep. (Vitaceae) shoots uninfected and infected with Rafflesia (Rafflesiaceae) and potential applications for propagation
}

\author{
Jeanmaire Molina ${ }^{1}$ (1) - Dejan Nikolic ${ }^{2}$. Jashvanth Raaj Jeevarathanam ${ }^{1} \cdot$ Rinat Abzalimov $^{3}$. Eun-Jung Park ${ }^{4}$. \\ Ronniel Pedales ${ }^{5}$. Elmer-Rico E. Mojica ${ }^{6}$. Danilo Tandang ${ }^{7,8}$. William McLaughlin ${ }^{9} \cdot K^{2}$ le Wallick ${ }^{9}$ James Adams ${ }^{9}$. \\ Ari Novy ${ }^{10,11}$ • Susan K. Pell ${ }^{9} \cdot$ Richard B. van Breemen ${ }^{12} \cdot$ John M. Pezzuto ${ }^{4,13}$
}

Received: 9 July 2021 / Accepted: 12 November 2021 / Published online: 29 November 2021

(c) The Author(s) 2021

\begin{abstract}
Main conclusion Metabolites in Rafflesia-infected and non-infected Tetrastigma were compared which may have applications in Rafflesia propagation. Benzylisoquinoline alkaloids, here reported for the first time in Vitaceae, were abundant in non-infected shoots and may be a form of defense. In Rafflesia-infected shoots, oxylipins, which mediate immune response, were elevated.
\end{abstract}

\begin{abstract}
Endemic to the forests of Southeast Asia, Rafflesia (Rafflesiaceae) is a genus of holoparasitic plants producing the largest flowers in the world, yet completely dependent on its host, the tropical grape vine, Tetrastigma. Rafflesia species are threatened with extinction, making them an iconic symbol of plant conservation. Thus far, propagation has proved challenging, greatly decreasing efficacy of conservation efforts. This study compared the metabolites in the shoots of Rafflesia-infected and non-infected Tetrastigma loheri to examine how Rafflesia infection affects host metabolomics and elucidate the Rafflesia infection process. Results from LC-MS-based untargeted metabolomics analysis showed benzylisoquinoline alkaloids were naturally more abundant in non-infected shoots and are here reported for the first time in the genus Tetrastigma, and in the grape family, Vitaceae. These metabolites have been implicated in plant defense mechanisms and may prevent a Rafflesia infection. In Rafflesia-infected shoots, oxygenated fatty acids, or oxylipins, and a flavonoid, previously shown involved in plant immune response, were significantly elevated. This study provides a preliminary assessment of metabolites that differ between Rafflesia-infected and non-infected Tetrastigma hosts and may have applications in Rafflesia propagation to meet conservation goals.
\end{abstract}

Keywords Benzylisoquinoline alkaloid · Ex situ conservation $\cdot$ Holoparasite $\cdot$ LC-MS · Oxylipin

\section{Abbreviations \\ ASRC Advanced Science Research Center, City Uni- versity of New York \\ BIA Benzylisoquinoline alkaloid \\ LC-MS Liquid chromatography-mass spectrometry \\ PUFA Polyunsaturated fatty acids}

Communicated by Dorothea Bartels.

Jeanmaire Molina

jeanmaire.molina@liu.edu

Extended author information available on the last page of the article
SDA Stearidonic acid

USBG United States Botanical Garden

\section{Introduction}

Rafflesia (Rafflesiaceae, Malpighiales) is a genus of plants producing the world's largest flowers (Fig. 1), yet it is a holoparasite with no stems, roots or leaves, deriving all nutrients solely from its host vine, the genus Tetrastigma (Vitaceae; Nais 2001; Davis et al. 2007). Ironically, the giant-flowered Rafflesia produces minuscule threadlike endophytes interspersed within the vascular tissue of its host (Nikolov et al. 


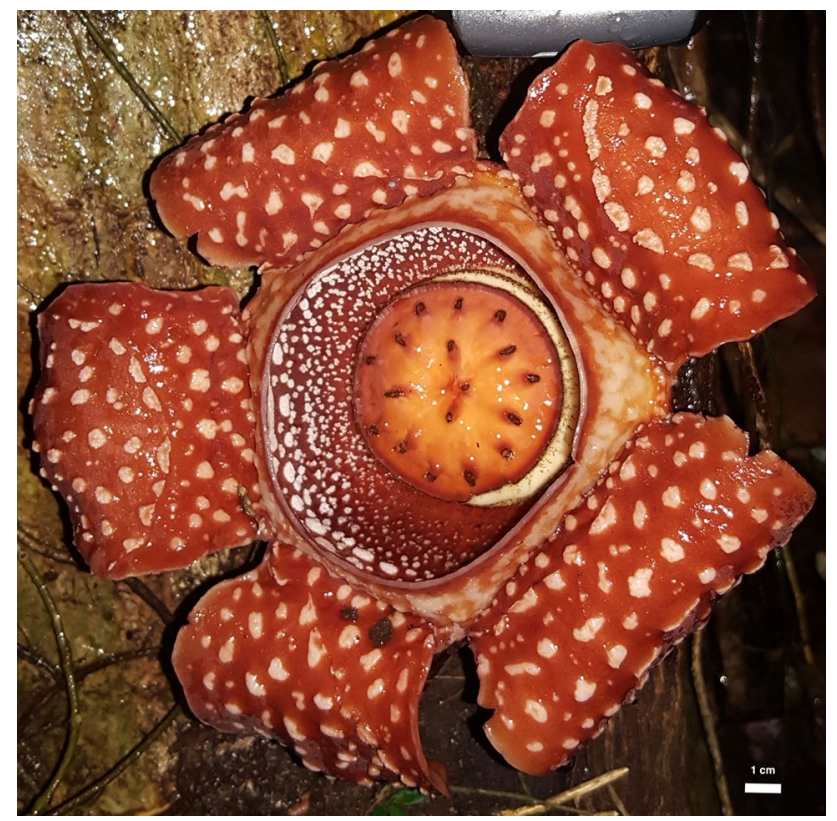

Fig. 1 Blooming flower of $R$. lagascae Blanco, the Rafflesia species infecting T. loheri Gagnep. (Mt Guinatungan, Camarines Norte, Philippines). This is one of the smaller Rafflesia species, at $20 \mathrm{~cm}$, with the largest ( $R$. arnoldii R.Br.) growing up to a meter. Photo by J. Molina

2014; Wicaksono et al. 2020). Dubbed the "panda of the plant world" for its charismatic characteristics, Rafflesia has ca. 30 species that are unique to the tropics of Southeast Asia with many endangered of extinction (Barcelona et al. 2009; Wicaksono et al. 2016). Unfortunately, efforts to propagate Philippine Rafflesia for ex situ conservation have had only incremental success (Molina et al. 2017). Molina and collaborators have been transporting Rafflesia-infected Tetrastigma cuttings from the Philippines for propagation at the United States Botanic Garden (USBG) in Washington, D.C. since 2015. The cuttings have rooted and survived for a maximum of 11 months, but no shoots have been produced.

Since Indonesia's Bogor Botanic Garden achieved blooms grafting Rafflesia-infected Tetrastigma to a Tetrastigma rootstock (Mursidawati et al. 2015; Wicaksono et al. 2016), this technique was replicated at the USBG. Unfortunately, the scion did not survive past 33 days. However, transported uninfected Tetrastigma cf. magnum Merr. and Tetrastigma harmandii Planch. seedlings survived at the USBG (Molina et al. 2017) and resulting mature plants have been repeatedly inoculated with $R$. speciosa seeds since Oct. 2017, but the emergence of Rafflesia buds has yet to be observed.

In holoparasitic Orobanchaceae, which includes Striga and other agricultural pests, a class of chemicals known as strigolactones produced by their host plants have been found to induce the parasitic plant's seed germination (Runyon et al. 2009; Smith et al. 2014). After germination, the Striga radicle grows toward host roots and forms a haustorium that allows the parasite to attach and obtain nutrients from the host. The haustorium is induced by host-derived small molecules (Albrecht et al. 1999; Saucet and Shirasu 2016) called haustorium-inducing factors (HIF) with 2,6-dimethoxy-p-benzoquinone (DMBQ) as the most active HIF for Striga (Wada et al. 2019). To determine if strigolactones also induce Rafflesia seed germination, Molina et al. (2017) incubated R. speciosa Barcelona \& Fernando seeds in GR24, a synthetic version of strigol. Other plant growth regulators were also investigated. However, none were able to stimulate Rafflesia seed germination (Molina et al. 2017). It is still unknown what host metabolites could facilitate a Rafflesia infection (Wicaksono et al. 2020), which is proving highly detrimental to conservation efforts.

There are limited studies on the chemical ecology of plant parasite-host interactions (Wink and Witte 1993; Loveys et al. 2001; Lozano-Baena et al. 2007; Runyon et al. 2009; Smith et al. 2009; Clarke et al. 2019; Furlan et al. 2019; Mutuku et al. 2020; Piwowarczyk et al. 2020). Since these interactions are metabolically diverse, involving two species of plants that may share biochemical characteristics (Lozano-Baena et al. 2007), chemical analysis is difficult (Allwood et al. 2008). Most studies of plant parasite-host interaction are on the parasitic taxa of Orobanchaceae and Cuscuta (Convolvulaceae) (Clarke et al. 2019; Mutuku et al. 2020). As agricultural pests, there has been a drive to understand their chemical ecology, such as germination stimulants (Runyon et al. 2009), to mitigate the economic impact of crop loss from these plant parasites. Other studies of these taxa (Lozano-Baena et al. 2007; Furlan et al. 2019) have examined the chemistry of host resistance to infection and determined that accumulation of phenolic compounds are toxic and suppressive to the parasite. An interplay between salicylates and jasmonates has also been shown to underlie effective plant defenses against insect herbivores, pathogens, and parasitic plants (Smith et al. 2009). Furlan et al. (2019) examined polyphenol content, which has also been implicated in plant defense, between Tapirira guianensis Aubl. trees (Anacardiaceae) parasitized and not parasitized by the mistletoe Phoradendron perrottetii (DC.) Eichler (Santalaceae), noting that parasite-infected tissues have less tannin/ polyphenol content than healthy tissues.

In this study, we aimed to compare the metabolites in Rafflesia-infected and non-infected Tetrastigma shoots to understand how Rafflesia infection affects host metabolomics. To our knowledge, this is the first study of its kind. A previous study of metabolites of Tetrastigma hemsleyanum Diels \& Gilg, a medicinal Chinese plant, but not a host of Rafflesia, identified constituent flavonoids, anthraquinones, esters, fatty acids, phenols, and catechins (Ding et al. 2019). Another T. hemsleyanum study elucidated the regulatory network of anthocyanin biosynthesis including 
metabolites involved in flavonoid biosynthesis and tryptophan metabolism, as well as alkaloids derived from the shikimate pathway (Yan et al. 2020). Because liquid chromatography-mass spectrometry (LC-MS)-based untargeted metabolomics has proven to be a quick, selective and highly sensitive method of analysis for a wide range of non-volatile metabolites (Commisso et al. 2013; Sargent 2013), we performed LC-MS to compare non-parasitized Tetrastigma shoots with infected shoots, to elucidate differences in host chemistry that could identify compounds useful in facilitating Rafflesia infection, and consequently, provide a new tool for conservation efforts.

\section{Materials and methods}

Cuttings of Rafflesia lagascae-infected Tetrastigma loheri Gagnep. and non-infected shoots were collected from San Lorenzo Ruiz Municipality, Mt. Guinatungan, Camarines Norte, Philippines, with permission from Mayor Nelson de Leon in May 2017 and in Aug 2018 (with Gratuitous permit no. 257 and 275 from the Philippine Biodiversity Management Bureau). The non-infected cuttings were taken from sufficiently mature woody host vines that did not have any visible sign of Rafflesia infection (i.e. Rafflesia floral buds/ scars absent), but mature enough that they could presumably support an infection, since Rafflesia has never been observed to infect juvenile vines. Our guide Ani Malate, who lived within the vicinity, has been tasked by the local government to regularly monitor Rafflesia populations for in situ conservation purposes, and is thoroughly experienced in determining which Tetrastigma plants were Rafflesia-infected. However, we were limited in sampling to ensure continued natural propagation of the Rafflesia and Tetrastigma populations and because of physical challenges in accessing various populations interspersed on mountainous terrain.

Samples were kept viable in moist sphagnum moss for about a week after collection during inspections and courier transport to USBG in Washington, DC (with USDA import permit P526P-18-02,136). Upon arrival at USBG, samples were placed in $-80{ }^{\circ} \mathrm{C}$ freezer until methanol extraction at LIU-Brooklyn (shipped from USBG overnight in dry ice). Samples from the cuttings were DNA-barcoded to determine the species of Tetrastigma following methods described previously (Molina et al. 2018).

Sections within ca. $5 \mathrm{~cm}$ of a Rafflesia bud, as well as comparable sections from non-infected cuttings ( 3 individuals for each type of cutting), were subjected to independent liquid chromatography mass spectrometry (LC-MS) experiments for confirmatory analyses, conducted at two different institutions: University of Illinois-Chicago College of Pharmacy (UIC) and the Advanced Science Research Center (ASRC) of the City University of New York. To control for differences due to sampling, comparable samples (i.e., same-age shoots) from infected and uninfected shoots were obtained. In both LC-MS runs, samples were first extracted in methanol ( $25 \mathrm{mg}$ ground in $700 \mu \mathrm{L}$ methanol) in July 2017 and in Nov 2018 following field collection. The extracts were evaporated to dryness under a gentle stream of nitrogen and then transported to UIC (July 2017, one set of infected and uninfected samples) and ASRC (Nov 2018, two sets of infected and uninfected samples). Samples were prepared for injection by reconstituting in $0.3 \mathrm{~mL}(\mathrm{v} / \mathrm{v})$ of $1: 1(\mathrm{v} / \mathrm{v}) \mathrm{MeOH} /$ water.

At UIC, samples were then analyzed using LC-MS with YMC AQ reverse phase column $2 \times 100 \mathrm{~mm}, 3 \mu \mathrm{m}$ ) and a Waters SYNAPT quadrupole/time-of-flight mass spectrometer operated in positive ion electrospray mode. A linear gradient was used from 10 to $90 \%$ acetonitrile in aqueous formic acid over $30 \mathrm{~min}$ at a flow rate of $0.2 \mathrm{~mL} / \mathrm{min}$ (column temperature $30^{\circ} \mathrm{C}$ ) with an injection volume of $5 \mu \mathrm{L}$. The mass spectrometric data were collected over the range $m / z$ 120-900.

At ASRC, samples were analyzed using a Bruker Daltonics maXis-II UHR-ESI-QqTOF mass spectrometer coupled to a Thermo Scientific Ultimate-3000 UHPLC system. Up to $20 \mu \mathrm{L}$ were injected onto an Agilent Acclaim 120 $\mathrm{C}_{18}$-column $(2.1 \mathrm{~mm} \times 100 \mathrm{~mm}, 5 \mu \mathrm{m})$ at $30{ }^{\circ} \mathrm{C}$ with a flow rate of $200 \mu \mathrm{L} / \mathrm{min}$. The gradient used was $0-1 \mathrm{~min} 7 \%$ solvent B (acetonitrile, $0.15 \%$ formic acid) and $93 \%$ solvent A (water, $0.15 \%$ formic acid) followed by a gradient $7-35 \% \mathrm{~B}$ from 1 to $15 \mathrm{~min}, 35-95 \%$ B from 15 to $28 \mathrm{~min}$, then held at $95 \%$ B from 28 to $31 \mathrm{~min}$. All experimental data were acquired over the range $m / z 50-1500$ using positive ion electrospray. The raw data were analyzed using the online version of XCMS metabolomics software (version 1.10.9; Tautenhahn et al. 2012). XCMS has been developed to facilitate an efficient workflow for untargeted metabolomics, which in contrast to targeted metabolomics, measures as many metabolites in the sample as possible. XCMS integrates metabolite profiling and identification in one step, including peak detection, retention time correction, chromatogram alignment and quantification (Benton et al. 2015). To analyze the data in XCMS, we applied a pairwise comparison between infected and non-infected samples with default parameters for Bruker Q-TOF (ASRC) with "bio-source = plant".

After XCMS analysis, the difference reports were filtered. XCMS integrates METLIN's high-resolution tandem mass spectrometry (MS/MS) database, which includes 1 million molecules including lipids, amino acids, carbohydrates, toxins, small peptides, and natural products, among other classes (Guijas et al. 2018). The features from XCMS with $P$ value $<0.05$, intensities above 20,000, and fold difference of at least 2.5, were analyzed further in Bruker Compass Data Analysis v4.3 and Metfrag Web (Ruttkies et al. 2016; https://msbi.ipb-halle. de/MetFragBeta/) to identify metabolites of interest. These 
parameters may be arbitrary but careful inspection of aligned peaks showed that these settings clearly distinguished the two groups providing a preliminary assessment of compounds that strongly differed between infected and non-infected hosts. We manually inspected each feature that passed our settings. Those that did not show a pronounced peak difference between the aligned extracted ion chromatograms (EIC) of features in infected and uninfected samples and/or those that were not annotated by METLIN were not included in further analysis.

The neutral molecular formula of the precursor ions (desired features) and their MS/MS fragmentation spectra were then obtained in Bruker Compass Data Analysis and given as input in the MS/MS peak list in Metfrag. All other settings were kept at default values. Candidate metabolites were then retrieved with the highest scoring candidates subjected to additional analysis in CFM-ID (Allen et al. 2014; http://cfmid. wishartlab.com/) to confirm Metfrag candidates. Metfrag and CFM-ID are silico fragmentation tools that utilize known compounds from structure databases to calculate fragments that are matched to experimentally obtained spectra (Blaženović et al. 2018). In addition to these automated approaches, we have also performed a manual dereplication approach on the data obtained from UIC to compare and verify the metabolites of interest from ASRC, as described in previous publications (Gödecke et al. 2009; Nikolić et al. 2012, 2015, 2017), some of which contain extensive analysis of fragmentation spectra of benzylisoquinoline alkaloids (BIA). Tandem mass spectra of BIAs and other metabolites identified in this study were compared to those stored in our in-house library and public databases (MoNA, Horai et al. 2010; GNPS, Wang et al. 2016).

\section{Data availability}

Experimental data from this study are publicly available in NIH Common Fund's National Metabolomics Data Repository (NMDR) website, the Metabolomics Workbench, https://www.metabolomicsworkbench.org, where it has been assigned Project ID PR001169. The data can be accessed directly via Project https://doi.org/10.21228/M8M40V Our data can also be accessed in XCMS including analyses to visualize extracted ion chromatograms (EIC), mass spectra, values of fold change, etc. of relevant features listed in Tables 1 and 2 upon request from corresponding author. In addition, quantitative aspects (fold-change, $P$ values, etc.) and corresponding EIC of relevant features presented in Tables S1 and S2 are available in the Supplement.

\section{Results}

DNA testing confirmed that 5 of 6 cuttings sampled were Tetrastigma loheri. One uninfected cutting was found to be T. papillosum (Blume) Planch., and LC-MS raw data for this sample were excluded from further analysis to rule out interspecific differences in metabolites. T. loheri is one of the six known Rafflesia host species in the Philippines (Pelser et al. 2019).

XCMS analysis yielded 14,457 metabolites/features within the range of retention time $0-35 \mathrm{~min}$. Upon filtering the LC-MS data, there were 422 (out of 14,457) features with $P$ values $<0.05$, intensities above 20,000 , and at least 2.5 -fold difference between infected and non-infected T. loheri. These settings clearly distinguished the aligned peaks of the two groups providing a preliminary assessment of significantly different metabolites.

Out of the 422 metabolites initially screened, a total of 18 could be further analyzed in Bruker Compass Data analysis, Metfrag and CFM-ID. Metabolites that did not show putative identification in METLIN were excluded. Tables 1 and 2 present metabolites significantly different between infected and non-infected hosts in both LC-MS runs. Since we performed two independent LC-MS analyses of the same samples, we are confident in the detection of these compounds. However, given our limited sampling and the XCMS filters we applied, there may be other compounds we may have missed, and our results are, therefore, preliminary.

Nine metabolites belonging to the class of benzylisoquinoline alkaloids were found to be significantly and naturally abundant in the non-infected T. loheri cuttings (Table 1). Identification of BIAs was based on the comparison with authentic standards, searches of public and in-house spectral databases and extensive prior knowledge on the fragmentation patterns of these compounds as described in several publications most notably those by Qing et al. (2020) and Menéndez-Perdomo et al. (2021). Figure 2 briefly summarizes structurally significant fragment ions that can be used to piece together a BIA molecule. Based on the masses of these fragment ions and by comparison with those of the known BIAs one can propose a reasonable structure of the unknown BIAs. For example, ion $\mathbf{c}$ representing loss of nitrogen determines whether nitrogen is present as a secondary amine (loss of $\mathrm{NH}_{3}$ ), tertiary amine (loss of $\mathrm{NH}-\mathrm{Me}$ ) or a quaternary amine (loss of $\left.\mathrm{N}\left(\mathrm{CH}_{3}\right)_{2}\right)$. Quaternary amines have an additional signature ion at $\mathrm{m} / \mathrm{z} 58$ corresponding to the $\left(\mathrm{CH}_{3}\right)_{2} \mathrm{~N}^{+}=\mathrm{CH}_{2}$ fragment formed by retro Diels-Alder fragmentation. Ion a on the other hand determines the substitution pattern on the benzyl side chain. For example, the $\mathrm{m} / \mathrm{z}, 107$ indicates $\mathrm{p}-\mathrm{OH}$ substituent, while $\mathrm{m} / \mathrm{z}, 121$ indicates p-OMe substituent. The structural arguments used to annotate a specific BIA are shown in Table 1.

On the other hand, eight metabolites were found to be significantly elevated in the Rafflesia-infected host compared to its non-infected counterpart (Table 2) including tryptamine, desoxypeganine, a pthalide (possibly cnidilide/neocnidilide), various kinds of polyunsaturated fatty acids (PUFA), and a polyphenol. 
Table 1 Metabolites significantly elevated in non-infected host. Comments including confidence levels following Schymanski et al. (2014) are provided

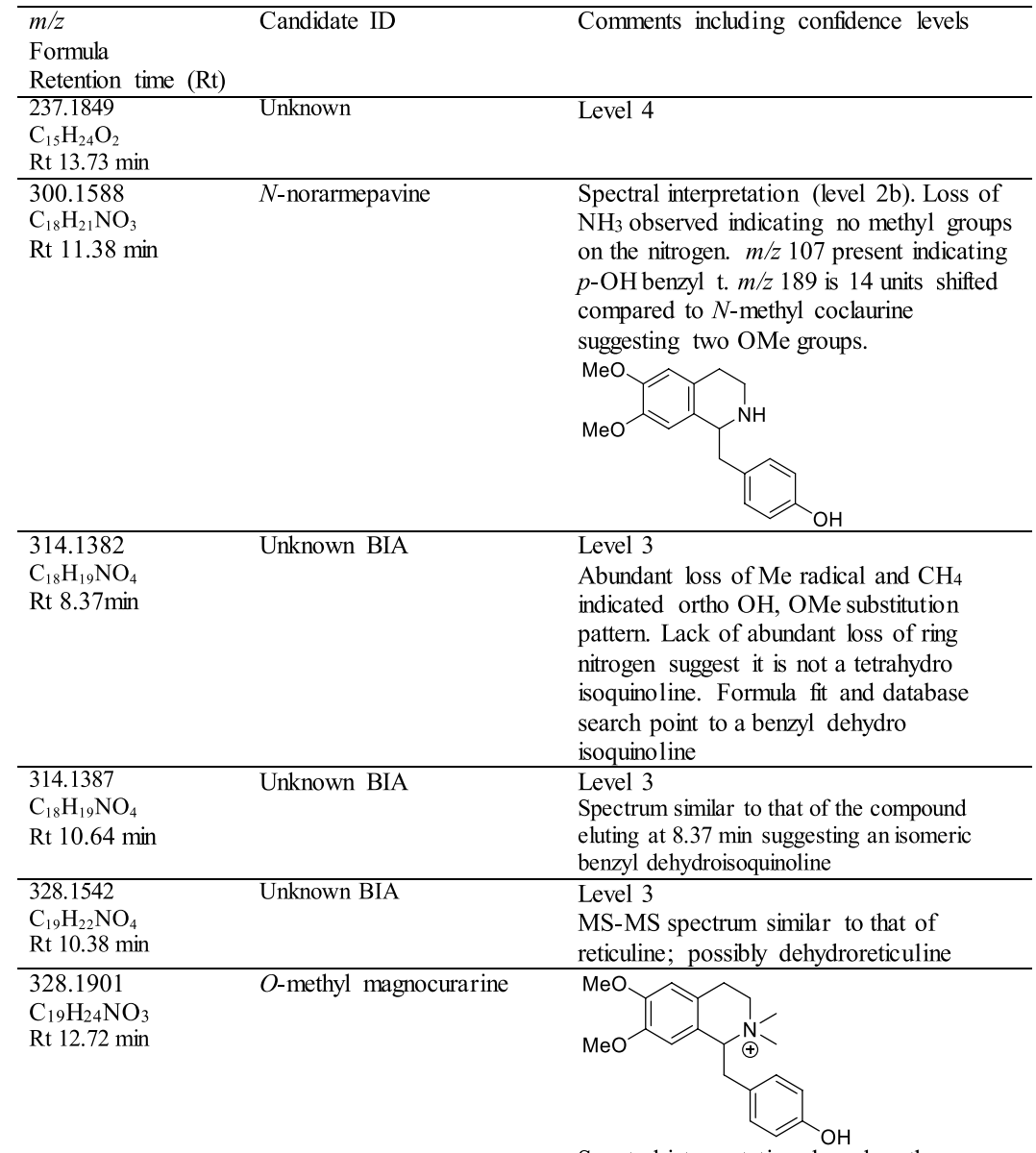

Spectral interpretation based on the tandem mass spectrum of magnocurarine (level 2b)

\begin{tabular}{lll}
\hline 330.1692 & Reticuline \\
$\mathrm{C}_{19} \mathrm{H}_{23} \mathrm{NO}_{4}$ & Standard (level 1)
\end{tabular}

Rt $9.79 \mathrm{~min}$

\begin{tabular}{|c|c|c|}
\hline 342.1699 & Magnoflorine & Standard (level 1) \\
\hline Rt $11.92 \mathrm{~min}$ & & \\
\hline 358.2008 & Quaternary BIA & Level 3 \\
\hline $\begin{array}{l}\mathrm{C}_{21} \mathrm{H}_{28} \mathrm{NO}_{4} \\
\mathrm{Rt} 11.24 \mathrm{~min}\end{array}$ & & $\begin{array}{l}\text { Molecular formula and loss of } \\
\text { dimethylamine point to a quaternary BIA. } \\
\text { Spectral info not sufficient for more } \\
\text { detailed structure proposal. }\end{array}$ \\
\hline 363.1797 & Secoisolariciresinol & Level2a \\
\hline $\begin{array}{l}\mathrm{C}_{20} \mathrm{H}_{26} \mathrm{O}_{6} \\
\text { Rt } 12.72 \mathrm{~min}\end{array}$ & & Spectral match \\
\hline
\end{tabular}


Table 2 Metabolites significantly elevated in infected host. Comments including confidence levels following Schymanski et al. (2014) are provided

\begin{tabular}{|c|c|c|}
\hline $\begin{array}{l}m / z \\
\text { Formula } \\
\text { Retention time (Rt) }\end{array}$ & Candidate ID & Comments including confidence levels \\
\hline $\begin{array}{l}144.0806 \\
\mathrm{C}_{10} \mathrm{H}_{9} \mathrm{~N} \\
\text { Rt } 8.29 \mathrm{~min} \\
\end{array}$ & Tryptamine & $\begin{array}{l}\text { Level 2a } \\
\text { Spectral match }\end{array}$ \\
\hline $\begin{array}{l}173.1119 \\
\mathrm{C}_{11} \mathrm{H}_{12} \mathrm{~N}_{2} \\
\text { Rt } 21.60 \mathrm{~min} \\
\end{array}$ & Desoxypeganine & $\begin{array}{l}\text { Proposed structure based on manual } \\
\text { interpretation combined with databases searches } \\
\text { of known compound with this formula }\end{array}$ \\
\hline $\begin{array}{l}195.1375 \\
\mathrm{C}_{12} \mathrm{H}_{18} \mathrm{O}_{2} \\
\text { Rt } 20.15 \mathrm{~min}\end{array}$ & Neocnidilide, cnidilide & \\
\hline & & $\begin{array}{l}\text { Level 3. This is a reasonable structure proposed } \\
\text { based on comparison with the tandem mass } \\
\text { spectrum of ligustilide. }\end{array}$ \\
\hline $\begin{array}{l}277.2155 \\
\mathrm{C}_{18} \mathrm{H}_{28} \mathrm{O}_{2} \\
\text { Rt } 20.15 \mathrm{~min}\end{array}$ & Stearidonic acid & Level 3 \\
\hline $\begin{array}{l}293.2104 \\
\mathrm{C}_{18} \mathrm{H}_{28} \mathrm{O}_{3} \\
\text { Rt } 19.28 \mathrm{~min}\end{array}$ & $\begin{array}{l}\text { 9,10-EOT, 12-OPDA, } \\
\text { etherolenic acid }\end{array}$ & Level 3 \\
\hline $\begin{array}{l}295.2260 \\
\mathrm{C}_{18} \mathrm{H}_{30} \mathrm{O}_{3} \\
\text { Rt } 20.15 \mathrm{~min}\end{array}$ & $\begin{array}{l}\text { 17-hydroxylinolenic acid, } \\
\text { 13(s)-HOT/(9Z,11E,15Z)- } \\
(13 S)-13 \text {-hydroxyoctadeca- } \\
9,11,15 \text {-trienoic acid }\end{array}$ & Level 3 \\
\hline $\begin{array}{l}313.2365 \\
\mathrm{C}_{18} \mathrm{H}_{32} \mathrm{O}_{4} \\
\text { Rt } 20.15 \mathrm{~min}\end{array}$ & $\begin{array}{l}(8 E, 10 S, 12 Z)-10- \\
\text { hydroperoxyoctadeca- } 8,12- \\
\text { dienoate, }(9 Z, 11 E)-(13 S) \text { - } \\
\text { 13-hydroperoxyoctadeca- } \\
\text { 9,11-dienoic acid }\end{array}$ & Level 3 \\
\hline $\begin{array}{l}743.2009 \\
\mathrm{C}_{32} \mathrm{H}_{38} \mathrm{O}_{20} \\
\text { Rt } 10.78 \mathrm{~min} \\
\end{array}$ & $\begin{array}{l}\text { Quercetin-3-(2g- } \\
\text { xylosylrutinoside) }\end{array}$ & Level 3 \\
\hline
\end{tabular}

\section{Discussion}

We sought to determine which metabolites were significantly different between Rafflesia-infected and non-infected T. loheri to understand how Rafflesia infection affects host metabolomics, to characterize these unique metabolites, as well as assess their potential ecological roles to generate insights that can facilitate a Rafflesia infection to aid in conservation efforts.

The Orobanchaceae germination stimulant strigol may potentially be present in both infected and uninfected Tetrastigma (results not shown) but was not significantly different and, therefore, excluded in the filter. Similar ligninrelated compounds that stimulate haustoria in Phelipanche and Striga (Orobanchaceae, Cui et al. 2018) were also detected in a METLIN search implemented within XCMS, in both Tetrastigma samples. However, it is unknown if they are involved in any way in facilitating a Rafflesia infection. The absence of germination when Rafflesia seeds were exposed to the synthetic strigol GR24 (Molina et al. 2017) suggests that there remain unknown aspects of Rafflesia's germination ecology (Wicaksono et al. 2020).

There were 10 metabolites that were found to be more abundant in non-infected T. loheri (Table 1) compared to 8 metabolites in Rafflesia-infected shoots (Table 2). Those with known ecophysiological roles in the literature are discussed below. We reiterate that these are compounds that passed our XCMS filters and manual dereplication approach, and there could be other compounds significantly different that have yet to be explored. Nonetheless, our study provides a critical first assessment of compounds that differed between infected and non-infected hosts so we may begin to understand how Rafflesia infection affects host metabolomics. It is possible that what we have determined to be "non-infected" hosts may be harboring dormant Rafflesia infection (i.e., Rafflesia buds have not yet emerged, Bascos et al. 2021), but given our local guide's expertise and experience in making this determination, and 


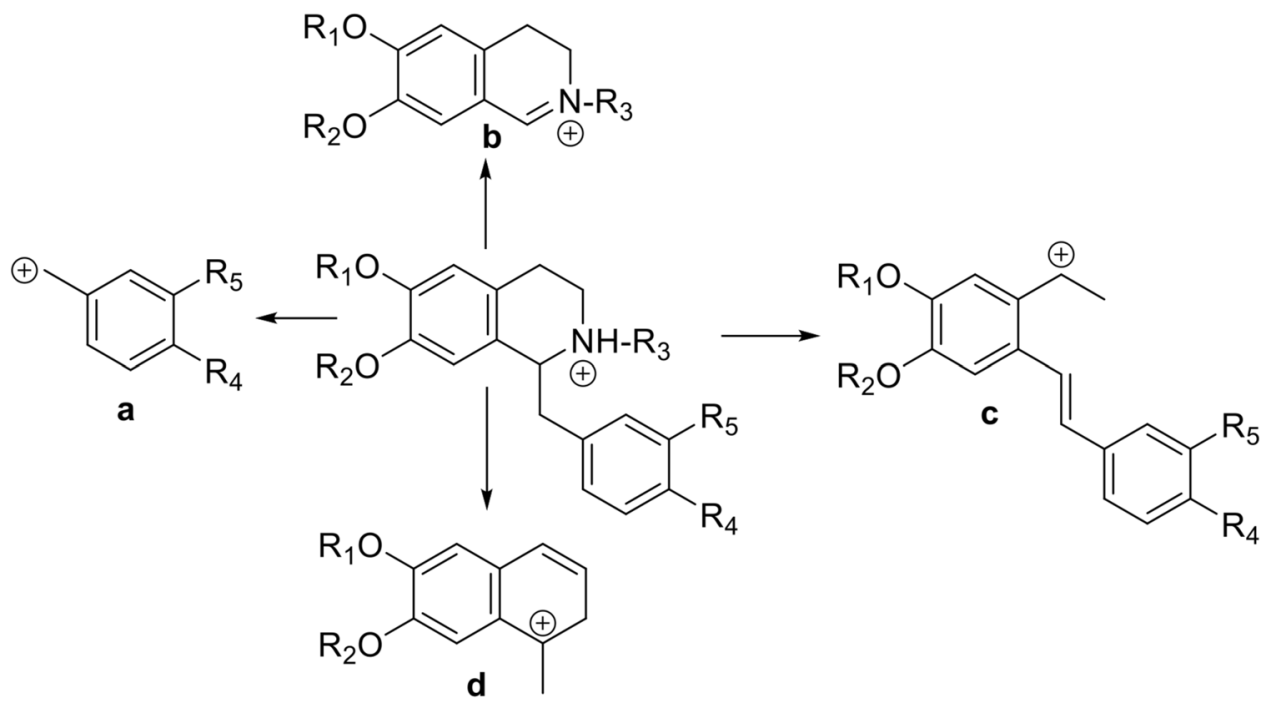

Fig. 2 Summary of fragmentation pattern of BIAs. Fragmentation scheme of BIAs used to identify compounds in this study. This summarizes structurally significant fragment ions that can be used to piece together a BIA molecule. Based on the masses of these fragment ions and by comparison with those of the known BIAs one can propose a reasonable structure of the unknown BIAs. For example, ion $\mathbf{c}$ representing loss of nitrogen determines whether nitrogen is present as a secondary amine (loss of $\mathrm{NH}_{3}$ ), tertiary amine (loss of

the low chance of unwittingly selecting three "dormant" samples, we are positive of the sampling, though additional sampling and analysis in the future would certainly be advantageous.

\section{Metabolites abundant in non-infected Tetrastigma Ioheri}

Benzylisoquinoline alkaloids (BIA) were significantly detected in non-infected $T$. loheri compared to infected shoots. BIAs are a diverse group of about 2,500 alkaloids that include pharmacologically important drugs such as codeine, morphine, tubocurarine, naturally produced in basal angiosperms (magnoliids including Annonaceae, Lauraceae and Piperales), as well as in the phylogenetically distant angiosperm order Ranunculales and infrequently, in the families Rutaceae, Cornaceae and Nelumbonaceae (Liscombe et al. 2005; Bonamore et al. 2010). BIA biosynthesis may have evolved as a mechanism for plant defense against herbivores and may have originated early on in angiosperm evolution, but its limited occurrence among angiosperms suggests the need for a "highly specialized cellular platform to activate the pathway in divergent taxa" (Liscombe et al. 2005: 2500). BIA is said to be absent in Eudicots (Cole et al. 2019), but our results imply that BIA may be sporadically present in eudicots and can be activated. Isoquinoline alkaloids are synthesized via decarboxylation of tyrosine or DOPA (dihydroxyphenylalanine) to yield dopamine and
NH-Me) or a quaternary amine (loss of $\left.\mathrm{N}\left(\mathrm{CH}_{3}\right)_{2}\right)$. Quaternary amines have an additional signature ion at $\mathrm{m} / \mathrm{z} 58$ corresponding to the $\left(\mathrm{CH}_{3}\right)_{2} \mathrm{~N}^{+}=\mathrm{CH}_{2}$ fragment formed by retro Diels-Alder fragmentation. Ion a on the other hand determines the substitution pattern on the benzyl side chain. For example, the $m / z, 107$ indicates p-OH substituent, while $\mathrm{m} / \mathrm{z}, 121$ indicates p-OMe substituent. The structural arguments used to annotate a specific BIA are shown in Table 1

4-hydroxyphenylacetaldehyde, which are then metabolized to reticuline, an important precursor of various BIAs. Substitution of the heterocycle isoquinoline at the $\mathrm{C} 1$ position by a benzyl group provides 1-benzylisoquinoline (Kanehisa and Goto 2000).

Though the pathway seems to have originated early on in the evolution of angiosperms, BIA production is only active in certain plant groups and is deactivated in others. However, this is the first time these compounds have been reported in Tetrastigma, and in the grape family, Vitaceae. As far as we know, no study has found BIA in the model species, Vitis vinifera $\mathrm{L}$., the common grape, which has been extensively characterized chemically (Pezzuto 2008; Pinu et al. 2018). However, enzymes involved in the initial steps of the pathway, prior to reticuline, are present in Vitis (Kanehisa and Goto 2000).

BIAs identified in non-infected T. loheri include reticuline, norarmepavine, magnocurarine, magnoflorine, and a few unknown BIAs (Table 1). In the BIA pathway, $(S)$-reticuline is the most common precursor for most BIAs formed from the methylation of methylcoclaurine. It is unclear how the other alkaloids are sequentially produced. Magnoflorine, magnocurarine have been identified in Magnolia officinalis Rehder \& E.H.Wilson (Poivre and Duez 2017). Armepavine, the methylated form of norarmepavine, on the other hand, is a major bioactive compound of Nelumbo nucifera Gaertn. and has been tested as a potential therapeutic agent for the treatment of a kidney disorder (Ka et al. 2010). 
Ironically, much more is known about the pharmacology of the BIAs (Singla et al. 2010), and there is little interest in their ecophysiological roles, which could provide insight as to why uninfected $T$. loheri may be producing these. BIAs generally do not appear essential for plant growth and development, but they play a key role in the plant defense against herbivores and pathogens (Hagel et al. 2013). Thus, in Tetrastigma loheri, these chemical adaptations may have evolved to fight off an infection including Rafflesia. In Genista anthoclada (Fabaceae) and its holoparasite Cuscuta palaestina (Convolvulaceae) several quinolizidine alkaloids were detected, with the alkaloids assumed to be exploited by Cuscuta for its own antiherbivore protection (Wink and Witte 1993). In addition to BIAs, the lignan, secoisolariciresinol, was also elevated in uninfected shoots. Lignans have potent antimicrobial and insecticidal properties and may have important roles in plant defense (Saleem et al. 2005).

Pennings and Callaway (2002) likened plant parasites to herbivores, as consumers with host preferences, but unlike animal herbivores, plant parasites are immobile and intimate with the host, and are thus more affected by host physiology and host biochemistry. Cuscuta seemed to have evolved to resist the toxic effects of the quinolizidine alkaloids and appropriate them for its own defense (Wink and Witte 1993), which does not seem to be the case in Rafflesia given that BIAs were found to be lacking in infected shoots. Nonetheless, it is also possible that unidentified microbial endophytes are producing the metabolites identified in Tetrastigma, as has been observed in opium poppy and in other plants (Ray et al. 2019).

Alternatively, Rafflesia could be suppressing the production of BIA in infected shoots. In terms of its application for ex situ propagation, T. loheri shoots with minimal BIA content may be more effective in supporting a Rafflesia infection and should be considered when grafting Rafflesia-infected shoots and when inoculating Rafflesia seeds.

\section{Metabolites elevated in Rafflesia-infected Tetrastigma loheri}

In Rafflesia-infected Tetrastigma shoots, there were eight metabolites that were found to be significantly elevated based on our XCMS settings. Noteworthy compounds include tryptamine, a phthalide (possibly cnidilide/neocnidilide) and various kinds of polyunsaturated fatty acids (PUFA) and the polyphenol quercetin-3-(2 g-xylosylrutinoside). The identification confidence for these are at level 3 (except tryptamine at 2a), meaning that the compound class (e.g. oxygenated fatty acids) is known, though the exact ID/ structure is unknown (Table 2) and have yet to be elucidated.

In the tryptamine pathway, the amino acid tryptophan is metabolized to tryptamine then ultimately to indole acetic acid, a bioactive auxin (Quittenden et al. 2009). Auxin signaling was found to be important in promoting xylem bridge formation between the Orobanchaceae hemiparasite Ptheroispermum japonicum and its host (Wakatake et al. 2020), and we speculate that the elevated trytamine would increase auxin that may forge vascular connections between Rafflesia and its host. Desoxypeganine was also found elevated in infected shoots but its ecological role is unknown, though it is pharmacologically characterized as a cholinesterase inhibitor (Algorta et al. 2008). The phthalide cnidilide (or neocnidilide) has been isolated as a flavor constituent in celery oil (MacLeod and Ames 1989). Interestingly, it is structurally related to seed-germination stimulants, strigol and karrikinolide (Renzetti and Fukumoto 2019), possessing a butenolide ring that may be biologically relevant (Fischer et al. 1989). Stearidonic acid is a PUFA naturally found in the seed oils of hemp and flaxseed (Bakowska-Barczak et al. 2020). Stearidonic acid (SDA) is derived from alpha-linolenic acid by a specialized enzyme, delta- 6 desaturase, not present in many plants (Ruiz-López et al. 2009). SDA is also important in human nutrition because it is an intermediate in the biosynthesis of eicosapentanoic acid (EPA) and docosahexaenoic (DHA) acids (Whelan et al. 2009). However, such as BIAs, its precise ecophysiological role is unclear, except that it is an intermediate product in the lipid pathways in some plants (Sreedhar et al. 2017). It is interesting to note, however, in the model nematode, Caenorhabditis elegans, its inability to produce gamma-linolenic acid and SDA due to loss-of-function mutation in the enzyme delta-6 desaturase led to increased pathogen susceptibility (Nandakumar and Tan 2008). Thus, the release of SDA in Rafflesia-infected T. loheri may be an immune response.

The PUFA hydroxy linolenic acid and 13(S)-HOT (9Z,11E,15Z)-(13S)-13-hydroxyoctadeca-9,11,15-trienoic acid) were also substantially increased in Rafflesia-infected shoots. These are types of oxygenated fatty acids, collectively termed 'oxylipins', which are involved in the immune response of plants (Genva et al. 2019). The plant hormone and oxylipin, jasmonate, is present ubiquitously in land plants playing a role in defensive responses (Griffiths et al. 2015). Like SDA, jasmonates are formed from linolenic acid in plant chloroplasts. Some oxylipins are distasteful to insect predators, and others can elicit a signal of cell damage throughout the plant to coordinate a comprehensive response (Gessler et al. 2017). Linolenic and linoleic acid production was also found to increase in tomato plants parasitized by the parasitic plant, Cuscuta pentagona Engelm., similar to the chemical response tomato plants display when attacked by herbivores or pathogens (Runyon et al. 2010). Fatty acid hydroperoxides (possibly $8 E, 10 S, 12 Z$ )-10-hydroperoxyoctadeca-8,12-dienoate and $(9 Z, 11 E)-(13 S)$-13-hydroperoxyoctadeca-9,11-dienoic acid), which serve as important 
intermediates in the oxylipin pathway (Hamberg et al. 1999), were also detected.

Another type of oxylipin detected in Rafflesia-infected shoots was a divinyl ether fatty acid (possibly 12-OPDA or etherolenic acid), which is similarly derived from alphalinolenic acid from the action of plant lipoxygenases (Fammartino et al. 2007; Vincenti et al. 2019). Though the physiological importance of divinyl ether fatty acids is not fully understood, it was observed that levels of this metabolite increased in infected potato leaves suggesting a possible role in defense response. Based on studies conducted with other plants, it is possible these oxylipins are released by $T$. loheri as a defense mechanism during Rafflesia infection. Thus, suppression of oxylipins in Rafflesia-infected Tetrastigma shoots may be beneficial in facilitating Rafflesia propagation.

Phenolics such as flavonoids and tannins have been demonstrated to be involved in plant defense against plant parasites (Lozano-Baena et al. 2007; Furlan et al. 2019). One flavonoid elevated in infected $T$. loheri was quercetin 3-(2 g-xylosyl rutinoside), but its ecological importance is so far not understood, yet multiple studies extol its dietary benefits (Anand David et al. 2016; Salehi et al. 2020). Piwowarczyk et al. (2020) detected abundant polyphenols, such as quercetin derivatives in the host species of the holoparasite Cistanche armena (K. Koch) M.V. Agab (Orobanchaceae). In grapes, quercetin glycosides as well as other flavonoids were associated with phytoplasma and mildew infection (Bouderias et al. 2020). Whether these polyphenols are involved in Tetrastigma immune reponse against its Rafflesia parasite remains to be seen.

\section{Conclusion}

In this study we provided a preliminary assessment of metabolites significantly different between Rafflesia-parasitized and non-parasitized Tetrastigma loheri. The abundance of benzylisoquinoline alkaloids (BIA) in non-infected host shoots suggest this metabolite may represent a defense strategy against Rafflesia infection, or that Rafflesia could somehow repress BIA production in infected shoots, where BIAs were shown to be lacking. The presence of BIA, a class of medicinally important compounds, in Tetrastigma and in its family Vitaceae, is here reported for the first time and reflects the pharmacological potential of this genus. Secretion of polyunsaturated fatty acids, of oxylipins, and polyphenols in Rafflesia-infected shoots, suggest that Rafflesia elicits host immune response. Conceivably, suppression of these immune-response compounds could facilitate Rafflesia infection and hence propagation. Further studies to test the metabolites identified here are the logical next steps to develop propagation strategies that could prove integral for preservation of this "panda of the plant world."

Supplementary Information The online version contains supplementary material available at https://doi.org/10.1007/s00425-021-03787-x.

Acknowledgements We are immensely grateful to the Philippine Biodiversity Management Bureau for their continued support, most especially to Dr. Mundita Lim, Nermalie Lita, Anson Tagtag, and Dr. Josefina de Leon. We thank Mayor Nelson de Leon, Engr. Rico Brizo, Bgy. Capt. Augrorio Adorino, PENRO's Marizon Vega and Maria Paz Yanto for allowing us to collect samples and our field guide Ani Malate. We thank RD Lourdes Wagan and Giselle of WRPSNCR for processing our export permits and the very accommodating staff of the NAIA Plant Quarantine office. We are also indebted to the supportive staff of the Philippine National Museum, esp. Dr. Ana Labrador, Dr. Tito Evangelista, John Rey Callado and Mike Galindon. We also extend our thanks to the reviewers of our manuscript, to Drs. Julie Barcelona, Ian Fontanilla, Joseph Morin, Jun Wen, Vicky Funk, Qing Cai, Perry Ong, and to all who have contributed one way or another to this project. This paper is dedicated to the memory of Leonard Co.

Author contributions JM conceived the project. JM, RP, DT, WM collected samples. JM, RA, DN, EJP performed the experiments. JM, JRJ, RA, DN, ERM analyzed the data. All authors contributed in writing the manuscript.

\section{Declarations}

Conflict of interests The authors declare no competing interests. The authors have no relevant financial or non-financial interests to disclose.

Open Access This article is licensed under a Creative Commons Attribution 4.0 International License, which permits use, sharing, adaptation, distribution and reproduction in any medium or format, as long as you give appropriate credit to the original author(s) and the source, provide a link to the Creative Commons licence, and indicate if changes were made. The images or other third party material in this article are included in the article's Creative Commons licence, unless indicated otherwise in a credit line to the material. If material is not included in the article's Creative Commons licence and your intended use is not permitted by statutory regulation or exceeds the permitted use, you will need to obtain permission directly from the copyright holder. To view a copy of this licence, visit http://creativecommons.org/licenses/by/4.0/.

\section{References}

Albrecht H, Yoder JI, Phillips DA (1999) Flavonoids promote haustoria formation in the root parasite Triphysaria versicolor. Plant Physiol 119:585-592. https://doi.org/10.1104/pp.119.2.585

Algorta J, Pena MA, Maraschiello C et al (2008) Phase I clinical trial with desoxypeganine, a new cholinesterase and selective MAO-A inhibitor: tolerance and pharmacokinetics study of escalating single oral doses. Methods Find Exp Clin Pharmacol 30:141-147. https://doi.org/10.1358/mf.2008.30.2.1159649

Allen F, Pon A, Wilson M et al (2014) CFM-ID: a web server for annotation, spectrum prediction and metabolite identification from tandem mass spectra. Nucleic Acids Res 42:W94-W99 
Allwood JW, Ellis DI, Goodacre R (2008) Metabolomic technologies and their application to the study of plants and plant-host interactions. Physiol Plant 132:117-135

Anand David AV, Arulmoli R, Parasuraman S (2016) Overviews of biological importance of quercetin: a bioactive flavonoid. Pharmacogn Rev 10:84-89. https://doi.org/10.4103/0973-7847. 194044

Bakowska-Barczak A, Larminat M, Kolodziejczyk PP (2020) The application of flax and hempseed in food, nutraceutical, and personal care products. In: Kozłowski RM, Mackiewicz-Talarczyk $M$ (eds) The textile institute book series, handbook of natural fibres, 2nd edn. Woodhead Publishing, pp 557-590

Barcelona JF, Pelser PB, Balete DS et al (2009) Taxonomy, ecology, and conservation status of Philippine Rafflesia (Rafflesiaceae). Blumea 54:77-93

Bascos EMA, Fernando ES, Duya MV et al (2021) Beginnings of a plant parasite: early development of Rafflesia consueloae inside its Tetrastigma host. Planta 254:61. https://doi.org/10.1007/ s00425-021-03710-4

Benton HP, Ivanisevic J, Mahieu NG et al (2015) Autonomous metabolomics for rapid metabolite identification in global profiling. Anal Chem 87:884-891. https://doi.org/10.1021/ac502 5649

Blaženović I, Kind T, Ji J et al (2018) Software tools and approaches for compound identification of LC-MS/MS data in metabolomics. Metabolites 8:31. https://doi.org/10.3390/metabo8020031

Bonamore A, Barba M, Botta B et al (2010) Norcoclaurine synthase: mechanism of an enantioselective pictet-spengler catalyzing enzyme. Molecules 15:2070-2078

Bouderias S, Teszlák P, Jakab G (2020) Age- and season-dependent pattern of flavonol glycosides in Cabernet Sauvignon grapevine leaves. Sci Rep 10:14241. https://doi.org/10.1038/ s41598-020-70706-7

Clarke CR, Timko MP, Yoder IJ et al (2019) Molecular dialog between parasitic plants and their hosts. Annu Rev Phytopathol 57:279299. https://doi.org/10.1146/annurev-phyto-082718-100043

Cole T, Hilger H, Stevens P (2019) Angiosperm phylogeny poster (APP) - Flowering plant systematics. https://peerjcom/preprints/2320 Accessed 31 July 2020

Commisso M, Strazzer P, Toffali K et al (2013) Untargeted metabolomics: an emerging approach to determine the composition of herbal products. Comput Struct Biotechnol J 4:e201301007. https://doi.org/10.5936/csbj.201301007

Cui S, Wada S, Tobimatsu Y et al (2018) Host lignin composition affects haustorium induction in the parasitic plants Phtheirospermum japonicum and Striga hermonthica. New Phytol 218:710 723. https://doi.org/10.1111/nph.15033

Davis C, Latvis M, Nickrent DL et al (2007) Floral gigantism in Rafflesiaceae. Science 315:1812

Ding F, Liu J, Du R et al (2019) Qualitative and quantitative analysis for the chemical constituents of Tetrastigma hemsleyanum Diels et Gilg using ultra-high-performance liquid chromatography/hybrid quadrupole-orbitrap mass spectrometry and preliminary screening for anti-influenza virus components. Evid-Based Compl Alt 2019:35-38. https://doi.org/10.1155/2019/9414926

Fammartino A, Cardinale F, Göbel C et al (2007) Characterization of a divinyl ether biosynthetic pathway specifically associated with pathogenesis in tobacco. Plant Physiol 143:378-388

Fischer N, Weidenhamer J, Bradow J (1989) Dihydroparthenolide and other sesquiterpene lactones stimulate witchweed germination. Phytochemistry 28:2315-2317

Furlan CM, Anselmo-Moreira F, Teixeira-Costa L et al (2019) Does Phoradendron perrottetii (mistletoe) alter polyphenols levels of Tapirira guianensis (host plant)? Plant Physiol Biochem 136:222-229
Genva M, Akong FO, Andersson MX et al (2019) New insights into the biosynthesis of esterified oxylipins and their involvement in plant defense and developmental mechanisms. Phytochem Rev 18:343-358

Gessler NN, Filippovich SY, Bachurina GP et al (2017) Oxylipins and oxylipin synthesis pathways in fungi. Appl Biochem Microbiol 53:628-639

Gödecke T, Lankin DC, Nikolic D et al (2009) Guanidine alkaloids and Pictet-Spengler adducts from black cohosh (Cimicifuga racemosa). J Nat Prod 72:433-437

Griffiths G (2015) Biosynthesis and analysis of plant oxylipins. Free Radical Res 49:565-582

Guijas C, Montenegro-Burke JR, Domingo-Almenara X et al (2018) METLIN: a technology platform for identifying knowns and unknowns. Anal Chem 90:3156-3164

Hagel JM, Facchini PJ (2013) Benzylisoquinoline alkaloid metabolism: a century of discovery and a brave new world. Plant Cell Physiol 54:647-672

Hamberg M, Sanz A, Castresana C (1999) $\alpha$-Oxidation of fatty acids in higher plants. J Biol Chem 274:24503-24513

Horai H, Arita M, Kanaya S et al (2010) MassBank: a public repository for sharing mass spectral data for life sciences. J Mass Spectrom 45:703-714. https://doi.org/10.1002/jms.1777

Ka S-M, Kuo Y-C, Ho P-J (2010) (S)-armepavine from Chinese medicine improves experimental autoimmune crescentic glomerulonephritis. Rheumatology 49:1840-1851

Kanehisa M, Goto S (2000) KEGG: Kyoto encyclopedia of genes and genomes. Nucleic Acids Res 28:27-30

Liscombe DK, MacLeod BP, Loukanina N et al (2005) Evidence for the monophyletic evolution of benzylisoquinoline alkaloid biosynthesis in angiosperms. Phytochemistry 66:1374-1393

Loveys BR, Tyerman SD, Loveys BR (2001) Transfer of photosynthate and naturally occurring insecticidal compounds from host plants to the root hemiparasite Santalum acuminatum (Santalaceae). Aust J Bot 49:9-16

Lozano-Baena MD, Prats E, Moreno MT, Rubiales D et al (2007) Medicago truncatula as a model for nonhost resistance in legumeparasitic plant interactions. Plant Physiol 145:437-449

MacLeod G, Ames J (1989) Volatile components of celery and celeriac. Phytochemistry 28:1817-1824

Menéndez-Perdomo IM, Hagel JM, Facchini PJ (2021) Benzylisoquinoline alkaloid analysis using high-resolution Orbitrap LC-MS ${ }^{\mathrm{n}} . \mathrm{J}$ Mass Spectrom 56:e4683. https://doi.org/10.1002/jms.4683

Molina J, McLaughlin W, Wallick K et al (2017) Ex situ propagation of Philippine Rafflesia in the United States: challenges and prospects. Sibbaldia 15:77-96

Molina J, Sherpa C, Ng J et al (2018) DNA barcoding of online herbal supplements: crowd-sourcing pharmacovigilance in high school. Open Life Sciences 13:48-55

Mursidawati S, Ngatari N, Irawati I et al (2015) Ex situ conservation of Rafflesia patma Blume (Rafflesiaceae)—an endangered emblematic parasitic species from Indonesia. Sibbaldia 13:99-110

Mutuku JM, Cui S, Yoshida S et al (2020) Orobanchaceae parasitehost interactions. New Phytol: https://doi.org/10.1111/nph.17083

Nais J (2001) Rafflesia of the world. Sabah Parks, Borneo, Malaysia

Nandakumar M, Tan MW (2008) Gamma-linolenic and stearidonic acids are required for basal immunity in Caenorhabditis elegans through their effects on p38 MAP kinase activity. PLoS Genet 4:e1000273. https://doi.org/10.1371/journal.pgen.1000273

Nikolić D (2017) CASMI 2016: A manual approach for dereplication of natural products using tandem mass spectrometry. Phytochem Lett 21:292-296

Nikolić D, Gödecke T, Chen SN et al (2012) Mass spectrometric dereplication of nitrogen-containing constituents of black cohosh (Cimicifuga racemosa L.). Fitoterapia 83:441-460 
Nikolić D, Lankin DC, Cisowska T et al (2015) Nitrogen-containing constituents of black cohosh: chemistry, structure elucidation, and biological activities. Recent Adv Phytochem 45:31-75

Nikolov LA, Tomlinson PB, Manickam S (2014) Holoparasitic Rafflesiaceae possess the most reduced endophytes and yet give rise to the world's largest flowers. Ann Bot 114:233-242

Pelser PB, Nickrent DL, van Ee BW et al (2019) A phylogenetic and biogeographic study of Rafflesia (Rafflesiaceae) in the Philippines: Limited dispersal and high island endemism. Mol Phylogenet Evol 139:106555. https://doi.org/10.1016/j.ympev.2019.106555

Pennings SC, Callaway RM (2002) Parasitic plants: parallels and contrasts with herbivores. Oecologia 131:479-489

Pezzuto JM (2008) Grapes and human health: a perspective. J Agric Food Chem 56:6777-6784

Pinu FR (2018) Grape and wine metabolomics to develop new insights using untargeted and targeted approaches. Fermentation 4:92. https://doi.org/10.3390/fermentation4040092

Piwowarczyk R, Ochmian I, Lachowicz S et al (2020) Phytochemical parasite-host relations and interactions: a Cistanche armena case study. Sci Total Environ 716:137071. https://doi.org/10.1016/j. scitotenv.2020.137071

Poivre M, Duez P (2017) Biological activity and toxicity of the Chinese herb Magnolia officinalis Rehder E Wilson (Houpo) and its constituents. J Zhejiang Univ Sci B 18:194-214

Qing Z, Xu Y, Yu L et al (2020) Investigation of fragmentation behaviours of isoquinoline alkaloids by mass spectrometry combined with computational chemistry. Sci Rep 10:733. https://doi.org/10. 1038/s41598-019-57406-7

Quittenden LJ, Davies NW, Smith JA et al (2009) Auxin biosynthesis in pea: characterization of the tryptamine pathway. Plant Physiol 151:1130-1138. https://doi.org/10.1104/pp.109.14150

Ray T, Pandey SS, Pandey A et al (2019) Endophytic consortium with diverse gene-regulating capabilities of benzylisoquinoline alkaloids biosynthetic pathway can enhance endogenous morphine biosynthesis in Papaver somniferum. Front Microbiol 10:925. https://doi.org/10.3389/fmicb.2019.00925

Renzetti A, Fukumoto K (2019) Synthesis of phthalides and $\alpha$, $\beta$-butenolides by transition metal-catalyzed activation of $\mathrm{C}-\mathrm{H}$ bonds. Molecules 24:824

Ruiz-López N, Haslam RP, Venegas-Calerón M et al (2009) The synthesis and accumulation of stearidonic acid in transgenic plants: a novel source of 'heart-healthy'omega- 3 fatty acids. Plant Biotechnol J 7:704-716

Runyon JB, Mescher MC, Felton GW et al (2010) Parasitism by Cuscuta pentagona sequentially induces JA and SA defence pathways in tomato. Plant Cell Environ 33:290-303

Runyon JB, Tooker JF, Mescher MC, et al (2009) Parasitic plants in agriculture: chemical ecology of germination and host-plant location as targets for sustainable control: A review. In: Lichtfouse E (eds) Organic farming, pest control and remediation of soil pollutants. Sustainable Agriculture Reviews, vol 1. Springer, Dordrecht. https://doi.org/10.1007/978-1-4020-9654-9_8

Ruttkies C, Schymanski EL, Wolf S et al (2016) MetFrag relaunched: incorporating strategies beyond in silico fragmentation. J Cheminform 8:3. https://doi.org/10.1186/s13321-016-0115-9

Saleem M, Kim HJ, Ali MS et al (2005) An update on bioactive plant lignans. Nat Prod Rep 22:696. https://doi.org/10.1039/b514045p

Salehi B, Machin L, Monzote L et al (2020) Therapeutic potential of quercetin: new insights and perspectives for human health. ACS Omega 5:11849-11872

Sargent M (2013) Guide to achieving reliable quantitative LC-MS measurements, RSC Analytical Methods Committee. ISBN
978-0-948926-27-3. https://www.rsc.org/images/AMC\% 20LCMS\%20Guide_tcm18-240030.pdf

Saucet SB, Shirasu K (2016) Molecular parasitic plant-host interactions. PLoS Pathog 12:e1005978. https://doi.org/10.1371/journ al.ppat. 1005978

Schymanski EL, Jeon J, Gulde R et al (2014) Identifying small molecules via high resolution mass spectrometry: communicating confidence. Environ Sci Technol 48:2097-2098

Singla D, Sharma A, Kaur J et al (2010) BIAdb: a curated database of benzylisoquinoline alkaloids. BMC Pharmacol 10:4. https://doi. org/10.1186/1471-2210-10-4

Smith SM (2014) Q\&A: What are strigolactones and why are they important to plants and soil microbes? BMC Biol 12:19. https:// doi.org/10.1186/1741-7007-12-19

Smith JL, De Moraes CM, Mescher MC (2009) Jasmonate- and salicylate-mediated plant defense responses to insect herbivores, pathogens and parasitic plants. Pest Manag Sci 65:497-503

Sreedhar RV, Prasad P, Reddy LPA et al (2017) Unravelling a stearidonic acid-rich triacylglycerol biosynthetic pathway in the developing seeds of Buglossoides arvensis: A transcriptomic landscape. Sci Rep 7:10473. https://doi.org/10.1038/s41598-017-09882-y

Tautenhahn R, Patti GJ, Rinehart D et al (2012) XCMS Online: a webbased platform to process untargeted metabolomic data. Anal Chem 84:5035-5039

Vincenti S, Mariani M, Alberti J-C (2019) Biocatalytic synthesis of natural green leaf volatiles using the lipoxygenase metabolic pathway. Catalysts 9:873. https://doi.org/10.3390/catal9100873

Wada S, Cui S, Yoshida S (2019) Reactive oxygen species (ROS) generation is indispensable for haustorium formation of the root parasitic plant Striga hermonthica. Front Plant Sci 10:328. https:// doi.org/10.3389/fpls.2019.00328

Wakatake T, Ogawa S, Yoshida S, et al (2020) An auxin transport network underlies xylem bridge formation between the hemi-parasitic plant Phtheirospermum japonicum and host Arabidopsis. Development 147:dev187781. doi: https://doi.org/10.1242/dev.187781

Wang M, Carver JJ, Phelan VV et al (2016) Sharing and community curation of mass spectrometry data with global natural products social molecular networking. Nature Biotech 34:828-837. https:// doi.org/10.1038/nbt.3597

Whelan J (2009) Dietary stearidonic acid is a long chain (n-3) polyunsaturated fatty acid with potential health benefits. J Nutr 139:5-10

Wicaksono A, Mursidawati S, Sukamto LA et al (2016) Rafflesia spp: propagation and conservation. Planta 244:289-296

Wicaksono A, Mursidawati S, Molina J (2020) A plant within a plant: insights on the development of the Rafflesia endophyte within its host. Bot Rev: https://doi.org/10.1007/s12229-020-09236-w

Wink M, Witte L (1993) Quinolizidine alkaloids in Genista acanthoclada and its holoparasite, Cuscuta palaestina. J Chem Ecol 19:441-448. https://doi.org/10.1007/BF00994317

Yan J, Qian L, Zhu W et al (2020) Integrated analysis of the transcriptome and metabolome of purple and green leaves of Tetrastigma hemsleyanum reveals gene expression patterns involved in anthocyanin biosynthesis. PLoS ONE 15:e0230154. https://doi.org/10. 1371/journal.pone.0230154

Publisher's Note Springer Nature remains neutral with regard to jurisdictional claims in published maps and institutional affiliations. 


\section{Authors and Affiliations}

Jeanmaire Molina ${ }^{1}\left(10\right.$ ) Dejan Nikolic ${ }^{2}$. Jashvanth Raaj Jeevarathanam ${ }^{1} \cdot$ Rinat Abzalimov $^{3}$. Eun-Jung Park ${ }^{4}$. Ronniel Pedales ${ }^{5}$. Elmer-Rico E. Mojica ${ }^{6}$. Danilo Tandang ${ }^{7,8} \cdot$ William McLaughlin $^{9} \cdot$ Kyle Wallick $^{9}$. James Adams ${ }^{9}$. Ari Novy ${ }^{10,11} \cdot$ Susan K. Pell ${ }^{9} \cdot$ Richard B. van Breemen ${ }^{12} \cdot$ John M. Pezzuto ${ }^{4,13}$

1 Department of Biology, Long Island University, Brooklyn, NY, USA

2 College of Pharmacy, University of Illinois, Chicago, IL, USA

3 Biomolecular Mass Spectrometry Facility, Advanced Science Research Center, City University of New York, New York, NY, USA

4 College of Pharmacy, Long Island University, Brooklyn, NY, USA

5 Institute of Biology, University of the Philippines Diliman, Quezon City, Philippines

6 Department of Chemistry and Physical Sciences, Dyson College of Arts and Sciences, Pace University, New York, NY, USA
7 Philippine National Herbarium (PNH), Botany Division, National Museum of the Philippines, Manila, Philippines

8 Academia Sinica, National Taiwan Normal University, Taipei, Taiwan

9 United States Botanic Garden, Washington, DC, USA

10 San Diego Botanic Garden, Encinitas, CA, USA

11 Department of Anthropology, University of California-San Diego, San Diego, CA, USA

12 Department of Pharmaceutical Sciences, College of Pharmacy, Oregon State University, Corvallis, OR, USA

13 College of Pharmacy and Health Sciences, Western New England University, Springfield, MA, USA 